\title{
Hierarchical Model for the Evolution of Cloud Complexes
}

\author{
Néstor M. Sánchez D. ${ }^{1,2}$ and Antonio Parravano ${ }^{1}$ \\ ${ }^{1}$ Centro de Astrofísica Teórica, Facultad de Ciencias, \\ Universidad de Los Andes, Mérida, Venezuela. \\ ${ }^{2}$ Departamento de Física, Facultad Experimental de Ciencias, \\ Universidad del Zulia, Maracaibo, Venezuela.
}

Received __; accepted _ 


\begin{abstract}
The structure of cloud complexes appears to be well described by a tree structure (i.e., a simplified "stick man") representation when the image is partitioned into "clouds". In this representation, the parent-child relationships are assigned according to containment. Based on this picture, a hierarchical model for the evolution of Cloud Complexes, including star formation, is constructed. The model follows the mass evolution of each sub-structure by computing its mass exchange with its parent and children. The parent-child mass exchange (evaporation or condensation) depends on the radiation density at the interphase. At the end of the "lineage", stars may be born or die; so that there is a non-stationary mass flow in the hierarchical structure. For a variety of parameter sets the system follow the same series of steps to transform diffuse gas into stars, and the regulation of the mass flux in the tree by previously formed stars dominates the evolution of the star formation. For the set of parameters used here as a reference model, the system tends to produce IMFs that have a maximum at too high mass $\left(\sim 2 M_{\odot}\right)$, and also the characteristic times for evolution seem too long. We show that these undesired properties can be improved by adjusting the model parameters. The model requires further physics (e.g. allowing for multiple stellar systems and clump collisions) before a definitive comparison with observations can be made. Instead, the emphasis here is to illustrate some general properties of this kind of complex nonlinear model for the star formation process. Notwithstanding the simplifications involved, the model reveals an essential feature that will likely remain if additional physical processes are included. That is: the detailed behavior of the system is very sensitive to the variations on the initial and external conditions, suggesting that a "universal" IMF is very unlikely. When an ensemble of IMFs corresponding
\end{abstract}


to a variety of initial or external conditions is examined, the slope of the IMF at high masses shows variations comparable to the range derived from observational data. These facts suggest that the considered physical processes (phase transitions regulated by the radiation field) may play a role in the global evolution of molecular complexes.

Subject headings: ISM: structure — ISM: clouds — Stars: formation — Stars: mass function 


\section{Introduction}

There are both theoretical (Zuckerman \& Palmer 1974; Silk 1978; Miyama et al. 1984) and observational (Scalo 1985; Lada et al. 1991; Houlahan \& Scalo 1992 and references therein) evidences that the formation of stars in molecular complexes occurs by successive fragmentation in a hierarchical manner. In fact, the maps of close complexes reveal clumps and irregularities on all observed scales and, inclusive, reveal some fractal nature (Dickman et al. 1990; Falgarone et al. 1991), whose origin is an important unsolved problem (see, e.g., the review of Elmegreen 1993). These complex structures are very difficult to model because of the diversity of processes that are involved (i.e. MHD, complex chemical networks, radiative transfer in an inhomogeneous medium). Many advances in the comprehension of these complex objects have been achieved by sectioning the problem and by focusing the attention on some scale and a few processes. However, in the present work we choose a schematic global approach, in which the relationship between some processes occurring at different scales can be examined. When nonlinear processes are involved, these simple global models help to understand the response of the system to variations of initial and boundary conditions, and the sensitivity to variations of free parameters.

A way to schematize the structure of molecular complexes is to consider that they are made of smaller sub-structures frequently denominated as "clouds". The definition of a "cloud" is ambiguous in the sense that it depends on the molecular line used for mapping and on the choice of the threshold intensity that define the boundaries (Scalo 1990). The hierarchical structure of these complexes can be described by using the "structure tree analysis" introduced by Houlahan \& Scalo (1992), which consists in a "stick man" representation obtained by partitioning the image into clouds. In the tree construction, a cloud is the "child" of another (of lower density) if its boundary lies within its parent, and the "skeleton image" is build up by connecting parent to child clouds. Based on a simplified 
version of this representation we elaborate a model to simulate some aspects of the evolution of interstellar cloud complexes by computing the mass exchange between parent and child clouds in a system of five levels of hierarchy. At the end of the tree structure it is assumed that stars may born and die, affecting drastically the mass circulation in the "tree".

In $\S 2$ the model is described and the basic equations are introduced. The results are discussed in $\S 3$ and, finally, the main conclusions are summarized in $\S 4$.

\section{The model}

A mass exchange model in a hierarchical geometry is constructed assuming a simplified Houlahan \& Scalo (1992) description: that is: a) we kept only information on the lineage of sub-structures (the Houlahan \& Scalo "structure tree" includes information on the spatial positions as well, but we neglect spatial information in the present model), and b) it is assumed that there are only five kinds of clouds that could develop in the five predefined levels in the stick man representation (the Houlahan \& Scalo representation is obtained by successively thresholding the image at decreasing column densities and identifying "clouds" as areas of connected pixels at each grey level). These five levels represent, in ascending order of density: warm gas $(w g)$, neutral gas $(n g)$, low density molecular gas $(l m g)$, high density molecular gas $(h m g)$ and protostars $(p s)$. The clouds at each level are assumed to differ only by their mass and by the number and mass of sub-structures they contain. These idealizations obviously exclude many physical processes that are known to occur (i.e., diffusion, collisions, etc.), but they allow to perform the global study of this complex system. We assume that each cloud can exchange mass with its parent cloud and it may develop until ten interior sub-structures (hereafter children), except at the last level where only one star may born from each protostar (that is, formation of multiple star systems is excluded). When a star dies, it incorporates a fraction of its mass to the warm gas; the 
remaining fraction rests as a stellar remnant. The first level (warm gas) is subjected to an external radiation field $U_{o}$ and may accrete mass from the environment at a rate $A_{c}$. Figure 1 schematizes the mass flux in a two children tree structure, though a ten children structure is considered in this work (i.e. $10^{4}$ places to form stars). The ideal number of available places for developing children (the branching ratio) should be large enough to ensure that every cloud always has a free place to condense a child cloud if the conditions are given for it. However, computational limitations restrict considering large branchings. A branching ratio of 10 is a compromise between computational time and saturation. Even when saturation occurs in some substructures in typical runs, a small fraction $(\sim 10 \%)$ of the total places in the tree structure are occupied. On the other hand, Houlahan and Scalo (1992) derived a value of 10 for the average number of children for the Taurus complex, although it is an uncertain result because it depends on the chosen threshold intensities used to define the cloud boundaries and on the resolution of the maps.

Even if the mass exchange has a relatively low connectivity (parent $\leftrightarrow$ cloud $\leftrightarrow 10$ children), it depends on the radiation transfer in the whole tree, which has a very large connectivity.

Suppose that each cloud can exchange mass with its parent at a rate $\phi_{p}$, and with its $j$-th child at a rate $\phi_{j}$, then, the net mass exchange rate of the $i$-th cloud with mass $M_{i}$ is

$$
\frac{d M_{i}}{d t}=\phi_{p}(i)-\sum_{j} \phi_{j}(i)
$$

where $\phi>0(\phi<0)$ implies condensation (evaporation). Mass conservation implies that if cloud $B$ is a child of cloud $A$, then $\phi_{p}(B)=\phi_{j=B}(A)$. Then, we can schematize the mass 
exchange equations as:

$$
\begin{array}{ll}
M_{w g}(t+\Delta t)=M_{w g}(t)+\left(A_{c}-\sum_{j} \phi_{j}\right) \Delta t+\alpha \sum_{s} M_{s} \delta\left(t-t_{s}-t_{*}\left(M_{s}\right)\right) & \text { if level =1, } \\
M_{i}(t+\Delta t)=M_{i}(t)+\left(\phi_{p}-\sum_{j} \phi_{j}\right) \Delta t & \text { if level }=2,3,4, \\
M_{p s}(t+\Delta t)=M_{p s}(t)+\phi_{p} \Delta t-M_{p s} \delta\left(M_{p s}-M_{c r i}\right) & \text { if level = }=5 ;
\end{array}
$$

where $M_{s}$ represents the mass of the $s$-th star formed at the instant $t_{s}$ whose lifetime is $t_{*}\left(M_{s}\right)$, and $M_{c r i}$, the critical mass required for a protostar of mass $M_{p s}$ to form a star, is a function of the history of the mass flux $\phi_{p}$ inward to the protostar (see below). At the end of the life of a star it is assumed that a mass $\alpha M_{s}$ is returned to the warm gas phase, whereas the fraction $(1-\alpha) M_{s}$ is left as a stellar remnant. The dependence of $\alpha$ on the mass of the star is assumed to be (David et al. 1990) $\alpha=1-1.4 / M_{s}$ if $M_{s}>6 M_{\odot}$ and $\alpha=0.84-0.44 / M_{s}$ if $M_{s}<6 M_{\odot}$. However, this parameter has little influence on the behavior of the model because the mass in stars is a small fraction of the mass of the system.

The mass exchange rate $\phi$ plays a central role in the model. Many physical processes mediate to determine its value which depends mainly on the unbalance between cooling and heating rates as well as on the unbalance between formation and destruction rates of chemical species. These rates depend on the local state of the gas and on the external conditions. In particular, the far UV radiation received by the cloud is an important factor in determining the heating rate and the dissociating rate of molecular species. For a given chemical state, the thermal evolution of a gas depends mainly on the excess of heating or cooling. If the pressure remains constant and cooling dominates over heating, the gas temperature decreases and the density increases. However, during the thermal evolution, both the chemical state of the gas and the cooling and heating efficiencies vary; generally a denser and cooler thermo-chemical equilibrium is attained: that is, a phase transition is achieved. The dominating physical processes that controls the mass exchange 
rate $\phi$ are different for the various transitions between the phases considered in the model. As an example, for the transition between the warm and neutral phases, the relevant physical processes are the cooling by the collisional excitation of heavy ions, the heating by photoejection of electrons by dust, and the ionization of hydrogen by collisions and by cosmic rays. On the other hand, a relevant process for the transition between the neutral and the low density molecular phases is the photodissociation by UV radiation of molecular species such as $\mathrm{H}_{2}$ and $\mathrm{CO}$.

The present model only takes care of two variables: the mass $M_{i}$ of substructures and the radiation density $U_{i}$ at their surfaces. Therefore we are restricted to adopt a schematic dependence of $\phi$ that involves only the location of the cloud in the tree structure and these two variables. Within this restriction, we assume that the mass exchange rate $\phi_{p}\left(M_{i}, U_{i}\right)$ between the substructure $i$ (located at level $l$ and being the $j$-th brother) and its parent cloud (located at level $l-1$ ) can be approximated by the product

$$
\phi_{p}\left(M_{i}, U_{i}\right)=\phi_{l} F\left(U_{i}, l, j\right) G\left(M_{i}, l\right)
$$

The first term $\phi_{l}$ is assumed to depend only on the cloud level $l$ and represents the typical rate of condensation of a cloud of "type $l$ " contained in a parent cloud of "type $l-1 "$ when it has a typical mass $M_{o, l}$ and no radiation field is present (i.e. $\phi_{p}\left(M_{o, l}, 0\right)=\phi_{l}$, $F(0, l, j)=1$, and $\left.G\left(M_{o, l}, l\right)=1\right)$. The adopted values of $\phi_{l}$ in Table 1 correspond to characteristic times $t_{l}=M_{o, l} / \phi_{l}$ of $0.67,0.2,0.02$, and 0.2 Myr for $l=2,3,4$, and 5 respectively. It is to be noticed that the time to condense a cloud of mass $M_{o, l}$ is much longer that $t_{l}$ because $G\left(M_{i}, l\right)<<1$ when $M_{i}<<M_{o, l}$ (see below).

The second term takes into account the dependence of $\phi$ on the radiation density $U_{i}$ at the surface of a cloud located in the position $(l, j)$ in the tree structure. In eq. $3 F\left(U_{i}, l, j\right)$ is the only factor that may change sign. Function $F$ is assumed to be positive for low values of $U_{i}$ (i.e. mass transfer to cloud $i$ from its parent cloud) and negative for high values of $U_{i}$ 
(i.e. mass transfer from cloud $i$ to its parent cloud). The adopted form for $F$ is

$$
F\left(U_{i}, l, j\right)=\left(1-\frac{U_{i}}{U_{c r i}(l, j)}\right),
$$

where,

$$
U_{c r i}(l, j)=U_{l} / j^{a_{l}} .
$$

That is, $F$ is a linear function of $U_{i}$ which acts as a switch to allow evaporation or condensation depending on the ratio of the radiation density $U_{i}$ to a critical value $U_{c r i}(l, j)$. This critical value depends mainly on the level $l$ at which the cloud $i$ is located and, in a lesser degree, on the position occupied by cloud $i$ in relation to their brothers clouds (i.e. its $j$ number).

In practice, the direction of the phase transition between a diffuse state (the parent cloud) and a denser one (the child cloud) not only depends on the radiation density but also on the local state, on the composition of the gas, and on others sources of heating, ionization or dissociation. However, their combined effect can be included in $U_{c r i}(l, j)$ if the physical conditions in clouds of the same type $(l, j)$ are assumed to be identical. The values for $U_{l}$ in eq. 5 can be estimated from the typical values of the critical energy density for the transition between the "ISM phases". For example, for the typical ISM pressures and gas composition in the solar vicinity, the critical energy density $U_{2}$ (corresponding to the transition from the $w g$ to the first $n g$ child; $l=2$ and $j=1$ ), has been estimated to be $\sim 7 \times 10^{-17} \mathrm{erg} \mathrm{cm}^{-3} \AA^{-1}$ in the band between $912-1100 \AA$ (Spitzer 1978; Parravano 1988). This is the relevant band at which $U_{c r i}$ must be calculated because this radiation is the main source of heating in these two phases. Also, this radiation is the main responsible of the photodissociation and heating in the molecular phases. For the purposes of this model, all the values for $U_{l}$ can be normalized to the value $U_{2}=1$. To estimate $U_{l}$ for the other transitions, specific models of photodissociation regions can be used (Hollenbach 1990, and references therein). If $\tau_{l}$ is the typical optical depth for the far UV radiation measured 
from the $w g$-ng interphase to the interphase of the phase $l$, then $U_{l}$ can be estimated as $U_{l} \sim U_{2} \exp \left(-\tau_{l}\right)$. The values of $U_{l}$ in Table 1 correspond to $\tau_{3}=0.7, \tau_{4}=8.5$ and $\tau_{5}=9.2$. Then, to transform the warm gas into protostars, the clouds at each level must grow until their optical depth is large enough to attenuate the radiation below the corresponding critical value to permit the development of child clouds. The weak dependence of $U_{c r i}$ on $j$ (the child number) is introduced in order to account for spatial variations on the physical conditions inside parent clouds, such as the pressure and the opacity. The adopted values of $a_{l}$ in Table 1 correspond to a difference in $U_{c r i}$ between the first and the 10 -th brother of $2 \%$ for levels 2, 3, and 4, and a difference of $17 \%$ for level 5 . The chosen values of these small differences in $U_{c r i}$ for the brothers clouds are arbitrary, but they are necessary to avoid the simultaneous formation and the identical evolution of all the child clouds in a given parent cloud.

The third term in eq. 3 takes into account the dependence of $\phi$ on the area of the interphase between the cloud and its parent. The adopted form for $G$ is

$$
G\left(M_{i}, l\right)=\left(\frac{M_{i}}{M_{o, l}}+\delta\right)^{2 / 3}
$$

However, function $G$ is set to zero when the condensation is switch-on (i.e. $F>0$ ) but the parent cloud is empty. The $2 / 3$ exponent comes from the assumption that $\phi$ is proportional to the surface of a spherical cloud of uniform density. The term $\delta=10^{-3}$ is introduced in order to initiate cloud condensation when the parent has available mass. Notice from eqs. 3 and 6 that if the ratio $\phi_{l} /\left(M_{o, l}\right)^{2 / 3}$ is kept constant, the parameter $M_{o, l}$ has little effect on $\phi$.

The regulating term $F\left(U_{i}, l, j\right)$ couples the local mass flux with the stars and gas distributions in the whole system via the radiation transfer in the tree. Thus, the radiation field controls the mass exchange in the system and regulates the star formation process. There are three contributions $\left(U_{\text {in }}(i)+U_{\text {out }}(i)+U_{\text {ext }}(i)\right)$ to the radiation density $\left(U_{i}\right)$ in the $i$-th cloud: 
- First, the radiation density $U_{\text {in }}$ corresponding to the radiation coming from the stars living in the substructures that are contained by the cloud. It is calculated recursively beginning from the fourth level. Due to the fact that there is not a protostar containing any star, the fifth level is not taken into account, and the interphase radiation density for a cloud at the fourth level is assumed to be

$$
U_{i n}(i)=\frac{e^{-\tau_{i}}}{D_{l}} \sum_{s} R_{s}
$$

where $\tau_{i}$ is the optical depth of the cloud, $D_{l}$ represents the dilution factor, and $R_{s}=r_{o} M_{s}^{3.5}$ represents the radiation density associated to the star $s$ inside the $i$-th $h m g$ cloud. The optical depth is assumed to be $\tau_{i}=c_{o} n_{l}^{2 / 3} M_{i}^{1 / 3}$ (i.e, proportional to the cloud size), $n_{l}$ being the number density at the level $l$ (see Table 1 ). The parameters $r_{o}$ and $c_{o}$ are fixed respectively to $6.3 \times 10^{-3}$ and $1.3 \times 10^{-2}$ in the Reference Model (see below). The dilution factor is also assumed to depend only on the level as the ratio of the typical cloud surfaces at consecutive levels (i.e. $D_{l}=\left[\left(n_{l+1} M_{o, l}\right) /\left(n_{l} M_{o, l+1}\right)\right]^{2 / 3}$.

For the superior levels $(l=3,2,1), U_{i n}(i)$ is calculated by summing the radiation $U_{\text {in }}(j)$ of their children attenuated by the optical depth $\tau_{i}$ of the cloud and diluted by the factor $D_{l}$. This is,

$$
U_{i n}(i)=\frac{e^{-\tau_{i}}}{D_{l}} \sum_{j} U_{i n}(j)
$$

- Second, the radiation density $U_{\text {out }}$ corresponding to the radiation coming from the stars in the system that are not contained by the cloud. Obviously, $U_{\text {out }}(w g)=0$. For the clouds at the levels 2, 3, 4 and 5, $U_{\text {out }}$ can be calculated recursively beginning with the second level. That is,

$$
U_{\text {out }}(i)=\left[U_{\text {out }}(p)+\sum_{k} U_{\text {in }}(k)\right] \exp \left(-\tau_{p}\right)
$$

where indices $p$ and $k$ indicate that the variables are evaluated on the parent or on the brothers clouds, respectively. 
- Third, the attenuated external radiation $U_{\text {ext }}(i)=U_{o} \exp \left(-\tau_{o i}\right)$ that corresponds to the external radiation $U_{o}$ attenuated by the total optical depth $\tau_{o i}$ from the surface of $i$-th cloud to the surface of the system. In order to explore the dependence of the system evolution on the external radiation field $U_{o}$, it is useful to introduce the quantity $\tilde{U}=U_{o} \exp \left(-\tau_{o, w g}\right) / U_{c r i}(2,1)$, where $\tau_{o, w g}$ is the initial optical depth of the warm gas cloud and $U_{\text {cri }}(2,1)=U_{2}=1$ is the surface critical radiation for the first warm gas child. If $\tilde{U}>1$, there is no condensation of warm gas even to its first child, and therefore, an initially warm gas remains as it is forever. The cases where $\tilde{U}$ is below but close to unity are of particular interest because it is expected that real "wg clouds" start to develop internal structures when the external condition changes from $\tilde{U}>1$ to $\tilde{U}<1$. This change may be produced, for example, by a decrease of the external radiation field or an increase of the warm gas optical depth due to an increase of the system mass or the external pressure. However, abrupt changes, due for example to cloud collisions or shock fronts, may reduce $\tilde{U}$ drastically below unity.

The star formation process is very difficult to model because it involves many interrelated physical processes. Moreover, the basic mechanism that determines the mass of a star has not yet been clearly established (see, for example, the reviews of Shu et al. 1987 and Pudritz et al. 1997). In the present model, we suppose that stars determine their own masses through the action of stellar winds (Adams \& Fatuzzo 1996). The mass of the formed star can be calculated as a function of the formation time $\left(t_{f}\right)$, i.e., the time interval that the protostar has been accreting mass from the parent cloud until the stellar wind stops the infall. The dashed line in Figure 2 corresponds to the mass of the star formed versus the formation time when the Adams \& Fatuzzo's (1996) conditions for stopping the accretion are used, except that it is assumed that the smallest possible stellar mass is 
$0.1 M_{\odot}$. The smooth curve shows the simple functional form assumed in this model, i.e.,

$$
M_{c r i}=\frac{C}{t_{f}^{\beta}}+M_{m i n}
$$

where $C=100, \beta=11 / 4$ and $M_{\min }=0.1 M_{\odot}$. The dotted line in Figure 2 represents an

example of the mass evolution of a protostar that is accreting mass at a variable rate from its parent cloud. When $M_{p s}=M_{c r i}$, the wind from the protostar is assumed to stop the accretion and a star of mass $M_{s}=M_{p s}$ appears at the place of the protostar. Therefore, as the accretion rate increases, the mass of the formed star increases and the formation time decreases.

Finally, each star is assumed to live a time $t_{*}$ given by (David et al. 1990)

$$
\log t_{*}\left(M_{s}\right)=4.0-3.42 \log M_{s}+0.88\left(\log M_{s}\right)^{2}
$$

where $M_{s}$ is in solar masses and $t_{*}$ in Myr. When a star dies, the localization of that star remains free to form another star.

\section{Results}

Only some aspects of the evolutionary process that are expected to be present in a molecular complex have been schematically considered in the above model. Therefore, it makes no sense to fine-tune the parameters of the model to try to reproduce particular observations. Instead, the model may be used to learn about the general behavior of this kind of complex network of interactions and mass transfer, and how its behavior depends on the model parameters and on the initial and environmental conditions. Even if the model has been designed in the simplest possible way, it was necessary to introduce a relatively large quantity of free parameters associated to the physical properties of the five gas "phases". 
Before describing the results of the model it may be useful to advance that the behavior of the system is chaotic in the sense that its temporal evolution is very sensitive to small changes in the initial or external conditions. This chaotic behavior is due to the nonlinearity of the included processes and to the large number of relationships between the clouds into the tree structure. It seems improbable that the inclusion of additional processes in the model can result in the avoidance of this chaotic behavior. As will be seen, due to this chaotic behavior, the results point towards a "non-universal" Initial Mass Function.

As a reference, detailed results will be shown for the set of parameters given in Table 1, and a particular initial and boundary condition. Due to the chaotic behavior of the system the results for this Reference Model are not representative of the detailed behavior of the system. However, this Reference Model is useful to illustrate the general manner in which the system evolves. After presenting the Reference Model results, the effect of variations of model parameters are summarized. Finally, by changing the initial and external condition in the Reference Model, we try to quantify the magnitude and nature of variations in the IMF. It is to be noticed that in a review of results concerning the IMF derived from star counts, Scalo (1998) concludes that there are strong indications of IMF variations, which do not seem to correlate with obvious environmental conditions like metallicity or stellar

density. In this sense, Scalo (1998) suggests that theoretical IMF models should account for these variations.

\subsection{Results for the Reference Model}

For the set of parameters given in Table 1, Fig. 3 shows the evolution of the total mass in each level (note that there may be as much as $10^{l-1}$ structures in level $l$ ). The initial state corresponds to a totally warm gas cloud of mass $M_{o}=2 \times 10^{5} M_{\odot}$, a mass accretion $A_{c}=0$, and an external radiation $\tilde{U}=0.95$. It can be seen that the warm gas mass (long 
dashed line labeled " $w g$ ") decreases as the condensation of matter to the neutral gas phase progresses (dotted line labeled " $n g$ "). The structures in the low density molecular level (dotted-long dashed line labeled "lmg") begin to condense matter when the mass of neutral gas reaches $\sim 1.5 \times 10^{4} M_{\odot}$ at $t \sim 25 M y r$. This is the time at which the first $n g$ cloud (i.e. $l=2$ and $j=1$ ) has accumulated enough material to attenuate the external radiation field just below the critical value $U_{c r i}(3,1)$, allowing the beginning of the condensation of the first $l m g$ cloud. This time would be reduced if the parameters $\phi_{2}, c_{o}$ (proportional to the optical depth by unit column density), or $U_{3}$ are increased. When the low density molecular gas has reached a large enough mass $\left(\sim 3 \times 10^{4} M_{\odot}\right)$, the high density molecular gas (dotted line labeled " $h m g$ ") starts to increase its mass and, rapidly, it begins to condense protostars (dotted-short dashed line labeled " $p s$ ") at $t \sim 66.3 \mathrm{Myr}$. When the first stars (solid line labeled " $s$ ") are born, at $t \sim 69 M y r$, both the high density molecular gas and the protostars are greatly affected, showing a very irregular behavior associated with the rapid variation of the radiation density. The total stellar mass grows up to $\sim 7 \times 10^{2} M_{\odot}$ and afterwards remains more or less constant because the radiation density reaches a "critical" value at which further condensation is inhibited. The decrease of the radiation density associated to the death of stars is rapidly compensated by the formation of new stars that again increase the radiation density. The first star dies at $t \sim 95 \mathrm{Myr}$ and afterwards the mass in remnants (short dashed line labeled " $r$ ") increases continuously.

The corresponding stellar formation history is showed in Fig. 4 (the dots give the formation events of one or more stars of mass $M$ ). The fact that the model does not produce stars less massive than $\sim 5 M_{\odot}$ after $t \sim 120$ Myr may be understood in terms of mass accumulation in the intermediate levels. The radiation of the previously formed stars inhibits the condensation of matter from the upper levels to the denser ones, accumulating mass in the low density molecular gas level; but, when the radiation field abruptly decreases due to the death of stars, the protostellar mass rapidly grows allowing the formation of 
massive stars only. This suppression of formation of low mass stars at late times is a general property of the model; it remains even when the model parameters, the initial conditions and the external conditions are varied (see next section). It must be mentioned that in this model we are not considering supernova explosions, that could be an important mechanism of stimulation or inhibition of star formation due to its drastic effect on the evolution of the cloud complex.

Figures 5a-5f show how the IMF builds up in a plot of the number of stars per unit logarithmic mass interval. At $t=70 M y r$ (Fig. 5a), when the star formation process just began, the star masses range from 2.5 to $10 M_{\odot}$; after this first burst, less massive stars start to form (Figs. 5b-5e, see also Fig. 4) until $t=120$ Myr (Fig. 5f), when low mass star formation is inhibited. At this time, the IMF shows a maximum at $M_{\max } \sim 1.6 M_{\odot}$, and the line of the better fit for $1.6 \leq M \leq 10 M_{\odot}$ (showed in the Figure $5 \mathrm{f}$ like a dashed line) has a slope $\Gamma=-1.48 \pm 0.32$. The efficiency $\epsilon$, defined as the ratio of the mass transformed in stars to the initial system mass, is $\sim 0.43 \%$ at $t=120 \mathrm{Myr}$. But, if at this time, the efficiency is calculated as the ratio of the mass transformed in stars to the gas mass excluding the warm gas, then the efficiency rise to $\sim 1.2 \%$ (i.e. a factor of about 2.5 higher). The last way for calculating the efficiency of the star formation efficiency is closer to the way in which observers estimate this quantity. However, in the rest of the paper, we use the former definition of $\epsilon$ because it refers to the whole system. It must be noticed that for the set of parameters used in the Reference Model, the system produces an IMF that has a flattening around $1.6 M_{\odot}$ and then a turn over at lower masses, but observational low-mass IMF studies indicate that the IMFs continue to rise below $1 M_{\odot}$, just with a flatter slope (maybe -0.5). Also, the characteristic times for evolution seem too long. These undesired properties may be improved by adjusting the model parameters (see below). However, the present model is a very schematic one to try to compare it with specific observations. Beside these undesired features, which may be produced by the star 
formation condition and because multiple stellar systems, cloud coalescence, and expanding

shells have not been allowed, the global evolutive pattern does not disagree with the general idea that we expect for the evolution of an "unperturbed" molecular complex.

\subsection{Dependence on model parameters}

In order to learn how the results change when the parameters of the model are varied, in the "Reference Model" various of these parameters have been changed one by one. The parameters that affect more the response of the system are: $C$ (related to the ability of stellar winds to stop accretion), $t_{l}=M_{o, l} / \phi_{l}$ (related to characteristic time to condense mass from the level $l-1$ in absence of radiation), and $c_{o}$ (the assumed optical depth of a structure with $M_{i}=1 M_{\odot}$ and $\left.n_{l}=1 \mathrm{~cm}^{-3}\right)$.

When $C$ is increased the curve $M_{c r i}\left(t_{f}\right)$ in Fig. 2 shifts toward higher masses. Therefore, protostars may accrete mass during more time and the mean mass of the formed stars increases. Runs of the model with $C$ from $1 / 5$ to 5 times the reference value $(C=100)$ show that as $C$ is increased the maximum of the IMF function shifts from $\sim 1.0$ to $\sim 2 M_{\odot}$, the slope $\Gamma$, calculated in the range $1.6 \leq M \leq 10 M_{\odot}$, increases from $\sim-1.3$ to $\sim-0.6$, and the efficiency $\epsilon$ increases from $\sim 0.3$ to $\sim 3 \%$. The IMF for $C=500$ is shown in Fig. 6a. However, a small variation of $C$ produces in general a large variation of these "integral" quantities but they remain in the above ranges and with the described tendencies. These results show that it is difficult to develop intuition on this kind of model. One may suspect that an increase of $C$ would reduce the star formation efficiency. Because the mean mass of the formed stars increases, the radiation produced by unit mass in stars also increases. Therefore, one may expect a reduction of the critical mass in the stellar system in order to maintain the radiation density at the critical level for condensation. However, after examination of the results one learns that this effect is overcome by others like the increased 
lifetime of protostars, i.e., before the more massive protostar in a given $h m g$ cloud starts to shine as a star, its brothers can grow during a longer time. Additionally, massive stars die faster.

When the characteristic time $t_{l}=M_{o, l} / \phi_{l}$ is increased the process of condensation of structures at level $l$ slows. For example, a decrease of $\phi_{4}$ (the parameter that allows to vary the velocity of mass exchange between the $l m g$ and $h m g$ phases) decreases the mass suply to the $h m g$ structures. Runs for the Reference Model show that little mass is represed in the $h m g$ phase, therefore, a decrease of $\phi_{4}$ indirectly tends to slow the the grow rate of protostars. Then, the typical protostar grow curve $M_{p s}\left(t_{f}\right)$ in Fig. 2 tends to reduce its slope and consequently, the mean mass of stars decreases. Runs for $\phi_{4}$ reduced to $1 / 2$, $1 / 5$ and $1 / 10$ the reference value $\left(5 \times 10^{3}\right)$ show that the mean mass of stars progressively decrease while the IMF flattens and its maximum shifts toward low masses. For the extreme case $\phi_{4}=5 \times 10^{2}$ the IMF (Fig. $6 \mathrm{~b}$ ) does not show maximum at all and only low mass stars form $\left(<3 M_{\odot}\right)$.

When $c_{o}$ is increased the optical depth by unit column density in each level is increased in the same proportion. Therefore, this parameter affects drastically the radiative coupling in the system. In order to analyze the effect of variations of $c_{o}$ respect to the reference case it is useful to adjust the external radiation in such a way to maintain $\tilde{U}$ at the reference value 0.95 . When $c_{o}$ is set to half the reference value, the time that the system must wait to form stars is enlarged appreciably $(\sim 200 M y r)$ and the IMF is dominated by massive stars. Conversely, when $c_{o}$ is twice the reference value, the time to start star formation is reduced to $\sim 30$ Myr and low and intermediate mass stars dominate the IMF as shown in Fig. 6c. In some ways this is the most satisfactory model with respect to observations. The timescale is reasonable $(30 \mathrm{Myr})$ and the turnover in the IMF is at low masses.

Even when general trends in the system response can be established when a model 
parameter is varied, it is to be noticed that the detailed evolution of the system, and even the "integral quantities" such as the IMF and $\epsilon$, are very sensible to small variations.

\subsection{Dependence on initial and external conditions}

As initial condition for all the runs we have assumed that a mass $M_{o}$ is in the $w g$ phase and that all the remaining phases are empty. This is the most primitive initial condition in the sense that it is the configuration that takes more time to start the formation of stars. This is an unlikely situation because this homogeneous state hardly occurs in the ISM. However, this initial condition allows to see how the IMF builds up departing from a diffuse unstructured gas cloud. Figure 7 shows the resulting star formation histories for four values of $M_{o}$. The label on each panel indicates the initial mass of the $w g$ cloud in units of $10^{5} M_{\odot}$. The parameters used are those of the Reference Model except that for each $M_{o}$

the external radiation is adjusted in such a way to maintain $\tilde{U}$ at the reference value 0.95 . Note that as the initial mass increases the time between bursts decreases, and the mean mass of formed stars increases. Figure 8 shows the corresponding IMFs at 150Myr for the same initial masses used in Fig. 7. The variation of the slope $\Gamma$ of the IMF is evident in Fig. 9, where the results for 40 different initial masses are shown. The plotted values and errors are obtained by fitting a line in the range $1.6 \leq M \leq 10 M_{\odot}$ of the IMF at $150 M y r$.

On the other hand, molecular complex are subjected to a variety of external conditions. Moreover, during its evolution the external conditions are expected to vary (i.e. heat input, external pressure and mass exchange). However, it is assumed that these conditions remain constant during runs of the model. In any case, it is expected that the chaotic behavior of the system will remain and even increase by adding random variations of external conditions. 
Because mass exchange in the system is controlled by radiation, the external radiation $\tilde{U}$ plays a central role. As $\tilde{U}$ is decreased the general tendency is that the time between bursts decreases and the mean mass of formed stars increases. It must be noted that in some cases all the mass is evaporated to the warm gas phase in the period where only massive stars are formed. When the radiation field in many places is high enough to evaporate surrounding high density material, the opacity decreases and radiation can easily permeate the tree structure up to the low density levels. In this case, all the gas becomes warm and $U_{i n}(w g)+U_{e x t}(w g)>1$. To restart condensation it is necessary to wait until enough stars die to decrease the radiation field and the condition $U_{\text {in }}(w g)+U_{\text {ext }}(w g)<1$ is again satisfied.

Again, small changes on $\tilde{U}$ produce large changes in the system response as showed in Fig. 10 where the evolution of the mass distribution, the star formation histories and the corresponding IMFs are plotted for two close values of $\tilde{U}$. The efficiency of the star formation process as function of $\tilde{U}$ is showed in Figure 11. Again, small variations of $\tilde{U}$ produce large variations of efficiency of star formation, but as expected, $\epsilon=0$ for the threshold value $\tilde{U}=1$. The slope $\Gamma$ in the range $1.6 \leq M \leq 10 M_{\odot}$ of the IMF as function of $\tilde{U}$ is showed in Figure 12. The values of $\Gamma$ did not show a correlation with variations of the external radiation field, ranging its value from -0.5 to -2.0 about of a mean value $\Gamma \simeq-1.4$.

Finally, the statistics of the $\Gamma$ values for 80 runs (i.e., 40 varying the initial mass (Fig. 9) and 40 varying the external radiation field (Fig. 12)) is showed in Fig. 13. The variability of the model IMFs is evident and roughly agree with the variability derived in a compilation of observational data by Scalo (1998). 


\section{Conclusions}

A lot of theoretical work has been done on the IMF (see, for example, Nakano et al. 1995; Adams \& Fatuzzo 1996; Padoan et al. 1997; see also the review of Zinnecker et al. 1993). Many theoretical works have successfully obtained the desired power law IMF departing from predefined cloud core properties distributions based on numerical and observational results. Also, pure statistical or geometrical approaches to model the process of gravitational fragmentation have been attempted by several authors (e.g. Auluck \& Kothari 1954; Reddish 1962; Fowler \& Hoyle 1963; Larson 1972; Elmegreen \& Mathieu 1983; Di Fazio 1986, Elmegreen 1997). However, the basic principles that determine the IMF shape and its dependence on the initial and external conditions remain unclear. In a recent review of results concerning the IMF derived from star counts, Scalo (1998) concludes that there are strong indications of IMF variations, which do not seem to correlate with obvious environmental conditions like metallicity or stellar density. Even when various sources of uncertainties are present in the determination of the IMF, the results summarized in his Fig. 5 (where the IMF slope for the reviewed Milky Way and LMC clusters has been plotted as a function of the average value of $\log M$ ) indicate that there is no evidence for a clustering of points around the Salpeter value (-1.3). Instead, there are significant variations of the IMF index at all masses above $1 M_{\odot}$. In this sense, Scalo (1998) suggests that theoretical IMF models should account for these variations.

By using a hierarchical structure description, a mass exchange model has been constructed to simulate some aspects of the evolution of molecular complexes. The schematic global approach in this work is completely deterministic and has allowed us to shift the assumed initial conditions from the distribution of the properties in cloud cores to the initial state of a single diffuse warm cloud. Although many of the physical mechanisms that are known to operate in molecular complexes are missing or schematized, this kind of 
models may help to identify relevant ingredients and typical behaviors, and to study the relationship between the various scales involved. The model presented here can be seen as an example of how this kind of schematic systems can provide insight about the behavior of complex ISM structures.

Even when the model considers few physical processes, its hierarchical structure allows to follow the evolution of a large quantity of variables at various scales. In fact, for a 10 children structure tree of five levels, at each time step the model actualizes the mass and the radiation density of 11111 substructures, and the condition for the formation or death of stars in $10^{4}$ places is verified. Then, the results showed are only a tiny fraction of the information generated in a run of the model.

It would be happen that a fine tuning of the parameters would be necessary to obtain a "reasonable" evolution; i.e. a progressive condensation of diffuse phases into denser ones through the tree structure - star formation at few places - evaporation of dense structures close to these places - star formation at new places until the global radiation field inhibits star formation at any place - new episodes of star formation when the radiation field decreases due to the death of stars. However, the model is robust in the sense that when the basic parameters or the initial and external conditions are varied the behavior of the system maintains its general pattern. This robustness comes from a global regulation that is controlled by the radiation field; that is: condensation tends to be inhibited by the radiation from stars but stars form from condensations. Molecular complexes are common structures that occurs in a variety of circumstances and therefore the robustness of the model may be seen as a desirable feature. It should be mentioned that the model tends to produce an IMF that have a maximum at too high mass and also the characteristic times for evolution seem too long. These undesired properties may be improved by adjusting the model parameters as shown in Sec. 3.2. For example, increasing the optical depth 
parameter $c_{o}$ resulted in a reduced time scale for the star formation of only $30 \mathrm{Myr}$ and an IMF that peaked at lower mass than the standard model. The deficit of high mass stars in that model may reflect the need for an accounting of collisional coalescence in the present model. However, too many processes are obviated in the model to try to compare its results with observations. Additionally, it makes no sense to adjust the model parameters without inferring their values from observations. The condition for star formation plays an important role in determining the shape of the IMF, and therefore the undesirable features in the IMF may also be improved by modifying the Adams \& Fatuzzo's condition, and by allowing the possibility of formation of multiple stellar systems, coalescence, stellar winds, HII regions, and explosions.

Notwithstanding the simplifications involved, this deterministic model reveals an essential feature that likely will remain if additional physical processes are included. That is: The detailed behavior of the system is very sensitive to the variations on the initial and external conditions, suggesting that a "universal" IMF is very unlike. It is interesting to note that looking at the ensemble of results presented here, the slope $\Gamma$ of the IMF at high masses (Fig. 13) shows a distribution that shares some characteristics with that derived from observational data (Scalo 1998). Obviously, this conclusion refers to the IMF for a single star forming "cloud". When talking about the universality of the IMF it is necessary to specify the scale at which the IMF refers. When an average over an ensemble of star forming regions is used to construct the IMF, the differences for different ensembles tend to be reduced. Then studies that use this kind of average and point toward a "universal" IMF are not necessarily in contradiction with a "non-universal" IMF at the scale of single star forming regions (i.e. Scalo 1998, and this work). Although it is out of the scope of the present work, the model can be used to quantify the variation of the average IMF for an ensemble of runs when a model parameter is varied. 
Finally, the model produces a characteristic pattern of evolution that is reminiscent of some aspects expected to occur in real molecular complexes. This fact, together with the robustness of the model, suggests that the included physical processes (phase transitions regulated by the radiation field) may play a role in the global evolution of molecular complexes.

We are very grateful to the referee Prof. John Scalo for the many suggestions that helped us to put our model in a much better context. This work was supported by C.D.C.H.T. of the Universidad de Los Andes and by CONDES of the Universidad del Zulia, Venezuela. 


\section{REFERENCES}

Adams, F. C., \& Fatuzzo, M. 1996, ApJ, 464, 256.

Auluck, F. C., \& Kothari, D. S. 1954, Nature, 174,565.

David, L. P., Forman, W., \& Jones, C. 1990, ApJ, 359, 29.

Di Fazio, A. 1986, A\&A, 159, 49.

Dickman, R. L., Horvath, M. A., \& Margulis, M. 1990, ApJ, 365, 586.

Elmegreen, B. G., \& Mathieu, R. D. 1983, MNRAS, 203, 305.

Elmegreen, B. G. 1993, in Protostars and Planets III, ed. E. Levy \& J. Lunine (Tucson: Univ. Arizona Press), 97.

Elmegreen, B. G., 1997, ApJ, 486, 944.

Falgarone, E., Phillips, T. G., \& Walker, C. K. 1991, ApJ, 378, 186.

Fowler, W. A., \& Hoyle, F. 1963, Roy. Obs. Bull., 11, 1.

Hollenbach, D. J. 1990, in The Evolution of the Interstellar Medium, ed. L. Blitz (San Francisco: ASP), 167.

Houlahan, P., \& Scalo, J. 1992, ApJ, 393, 172.

Lada, E. A., Bally, J., \& Stark, A. A. 1991, ApJ, 368, 432.

Larson, R. B. 1972, Nature, 236,21.

Miyama, S. M., Hayashi, C., \& Narita, S. 1984, ApJ, 279, 621.

Nakano, T., Hasegawa, T., \& Norman, C. 1995, ApJ, 450, 183. 
Padoan, P., Nordlund, A., \& Jones, B. J. T. 1997, MNRAS, 288, 145.

Parravano, A. 1988, A\&A, 205, 71.

Pudritz, R. E., McLaughlin, D. E., \& Ouyed, R. 1997, preprint, astro-ph/9702232.

Reddish, V. C. 1962, Sci. Progr., 50, 235.

Scalo, J. M. 1985, in Protostars and Planets II, ed. D. C. Black \& M. S. Matthews (Tucson: Univ. of Arizona Press), 201.

Scalo, J. 1990, in Physical Processes in Fragmentation and Star Formation, Ed. R. CapuzzoDolcetta, C. Chiosi \& A. Di Fazio (Dordrecht: Kluwer Academic Publishers), 151.

Scalo, J. M. 1998, in The Stellar Initial Mass Function, proceedings of the 38th Herstmonceux Conference, ed. G. Gilmore, I. Parry, and S. Ryan (in press).

Shu, F. H., Adams, F. C., \& Lizano, S. 1987, ARA\&A, 25, 23.

Silk, J. 1978, in Protostars and Planets, ed. T. Gehrels (Tucson: Univ. Arizona Press), 172.

Spitzer, L. 1978, Physical Processes in the Interstellar Medium (New York: Wiley).

Zinnecker, H, McCaughrean, M. J., \& Wilking, B. A. 1993, in Protostars and Planets III, ed. E. Levy \& J. Lunine (Tucson: Univ. Arizona Press), 429.

Zuckerman, B., \& Palmer, P. 1974, ARA\&A, 12, 279. 
Fig. 1. - Schematic representation of mass flux in the tree (see text). For simplicity only two children for each cloud have been represented.

Fig. 2. - The critical mass for star formation $\left(M_{c r i}\right)$ as a function of the formation time $\left(t_{f}\right)$. The continuous line is the assumed functional form (eq. 10), the dashed line shows the condition obtained from Adams \& Fatuzzo (1996), and the dotted line shows a typical mass evolution of a protostar $\left(M_{p s}\right)$.

Fig. 3.- The evolution of the total mass of warm gas $(w g)$, neutral gas $(n g)$, low density molecular gas $(l m g)$, high density molecular gas $(h m g)$, protostars $(p s)$, stars $(s)$ and remnants $(r)$, for the Reference Model (see text) for $M_{o}=2 \times 10^{5} M_{\odot}$ and $\tilde{U}=0.95$.

Fig. 4.- Star formation events for the parameters values used in Figure 3.

Fig. 5.- The IMF at various times for the same parameter values used in Fig. 3. a) $t=70 \mathrm{Myr}, \mathrm{b}) t=80 \mathrm{Myr}, \mathrm{c}) t=90 \mathrm{Myr}, \mathrm{d}) t=100 \mathrm{Myr}, \mathrm{e}) t=110 \mathrm{Myr}$ and $\mathrm{f}$ ) $t=120$ Myr. The dashed line in Fig. 5 f shows the line of the better fit for $1.6 \leq M \leq 10 M_{\odot}$.

Fig. 6. - The IMF at $t=120 M y r$ when the parameters of the Reference Model are varied. a) when $C$ is five times greater than the reference value; b) when $\phi_{4}$ is ten times lower than the reference value; c) when $c_{o}$ is twice the reference value.

Fig. 7.- The star formation histories for four values of the initial mass. The parameters used are those of the Reference Model and $\tilde{U}=0.95$.

Fig. 8. - The IMF at $t=150$ for the same initial masses used in Fig. 7.

Fig. 9.- The slope of the IMF in the range $1.6 \leq M \leq 10 M_{\odot}$ as function of the initial mass for 40 model runs. The parameters used are those of the Reference Model and $\tilde{U}=0.95$.

Fig. 10.- The system response for two close values of $\tilde{U}$ when the parameters of the 
Reference Model are used, and $M_{o}=2 \times 10^{5} M_{\odot}$. a,b,c) For $\tilde{U}=0.96$, the evolution of the mass distribution, the star formation history, and the IMF at $t=150$, respectively. d,e,f) The same as in Fig. 10a,b,c but for $\tilde{U}=0.9625$.

Fig. 11. - The efficiency of the star formation process as function of $\tilde{U}$ for 40 model runs. The parameters used are those of the Reference Model and $M_{o}=2 \times 10^{5} M_{\odot}$.

Fig. 12. - The slope of the IMF in the range $1.6 \leq M \leq 10 M_{\odot}$ as function of $\tilde{U}$ for 40 model runs in Fig 11.

Fig. 13. - Histogram of the distribution of the IMF slopes in the range 1.6 to $10 M_{\odot}$ for 80 runs, i.e., 40 varying the initial mass (Fig. 9) and 40 varying the external radiation field (Fig. 12). 
Table 1. Parameters of the Reference Model

\begin{tabular}{cccccc}
\hline \hline Level $(l)$ & $n_{l}\left(\mathrm{~cm}^{-3}\right)$ & $M_{o, l}\left(M_{\odot}\right)$ & $\phi_{l}\left(M_{\odot} /\right.$ Myr $)$ & $U_{l}$ & $a_{l}$ \\
\hline & $2 \times 10^{-1}$ & $\ldots$ & $\ldots$ & $\ldots$ & $\ldots$ \\
1 & 6.6 & $1 \times 10^{4}$ & $1.5 \times 10^{4}$ & 1 & $8 \times 10^{-3}$ \\
2 & $2 \times 10^{2}$ & $1 \times 10^{3}$ & $5.0 \times 10^{3}$ & $5 \times 10^{-1}$ & $8 \times 10^{-3}$ \\
3 & $6 \times 10^{3}$ & $1 \times 10^{2}$ & $5.0 \times 10^{3}$ & $2 \times 10^{-4}$ & $8 \times 10^{-3}$ \\
5 & $1 \times 10^{5}$ & 1 & 5.0 & $1 \times 10^{-4}$ & $8 \times 10^{-2}$ \\
\hline
\end{tabular}




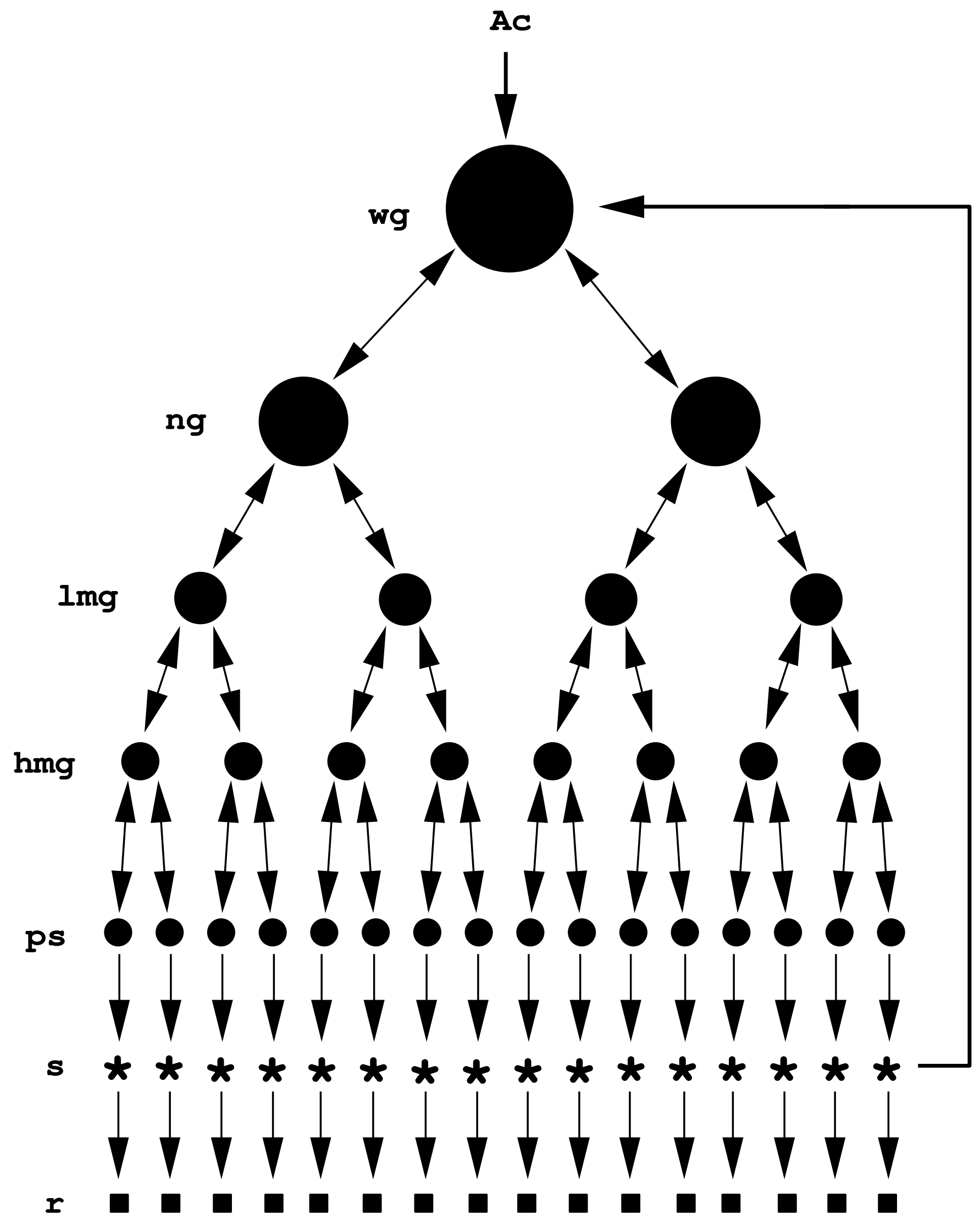




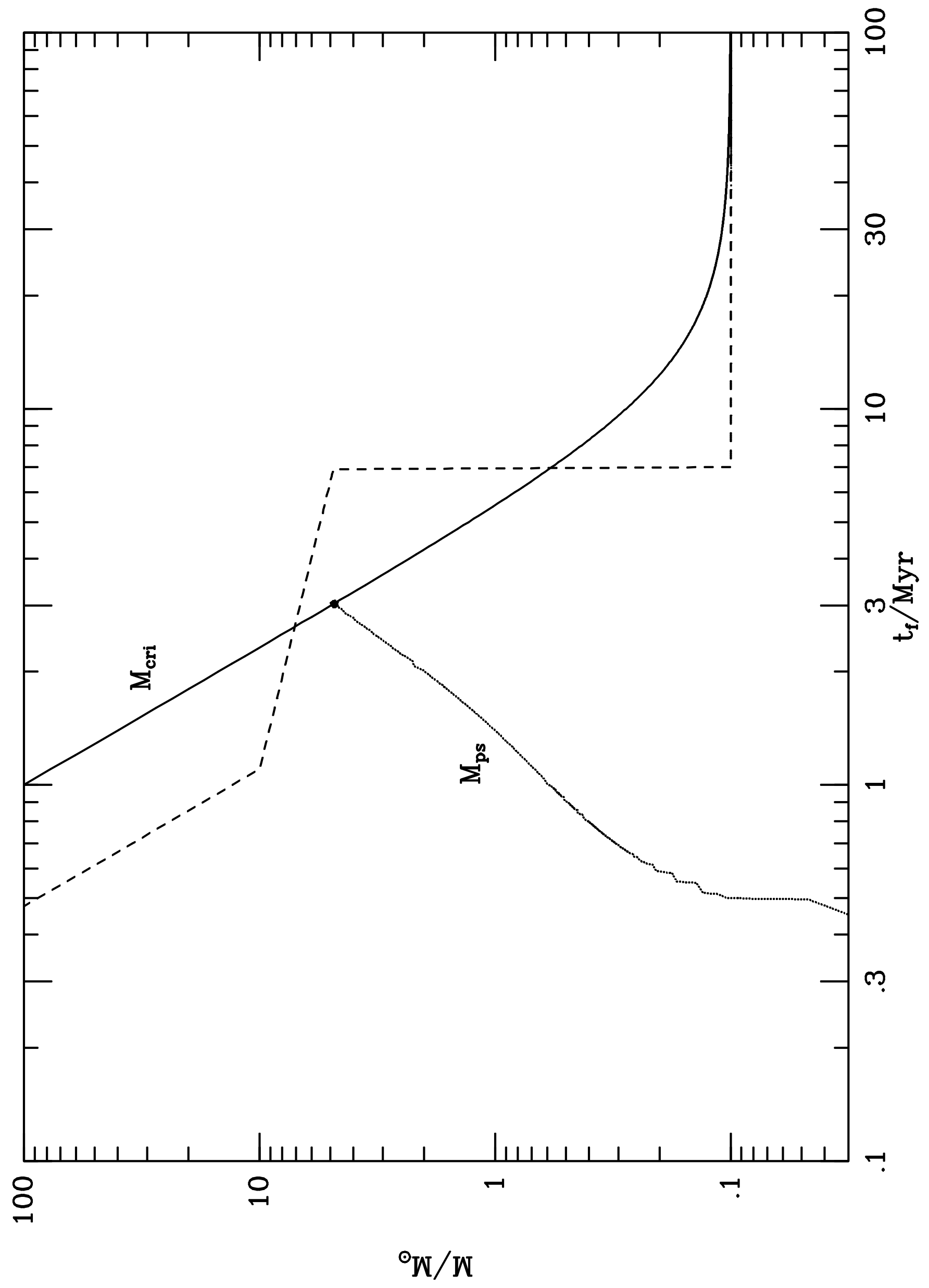




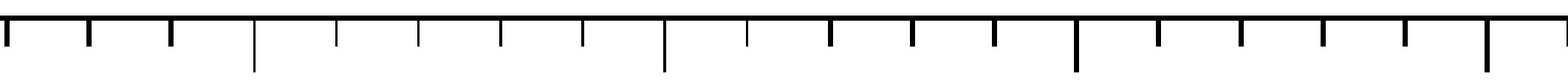
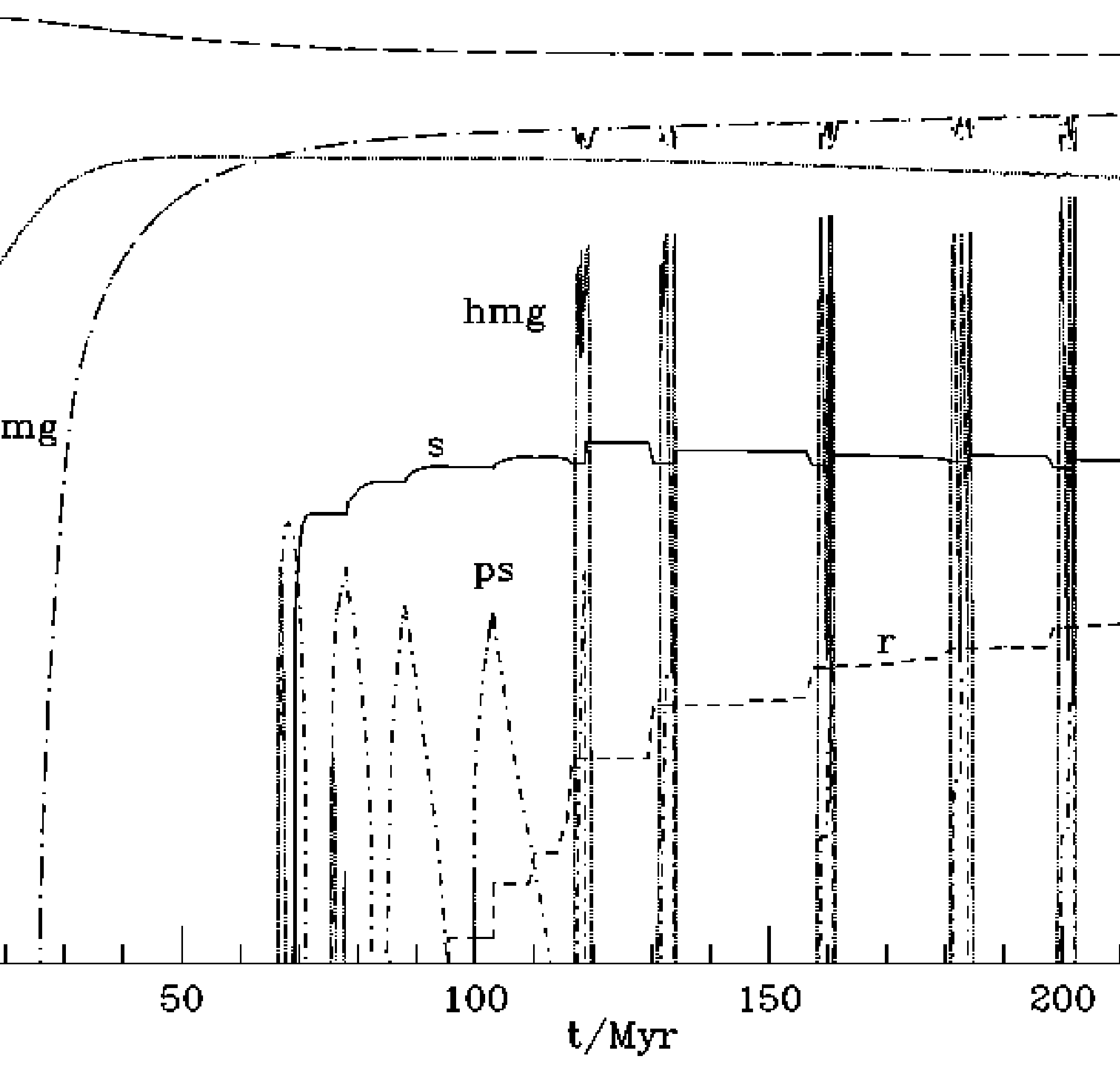


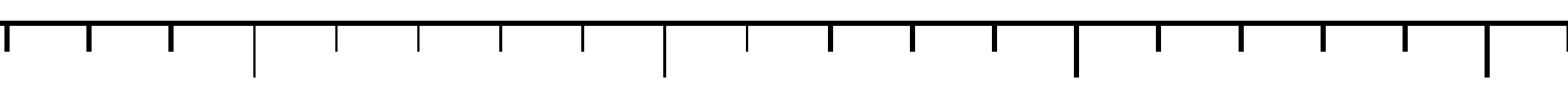




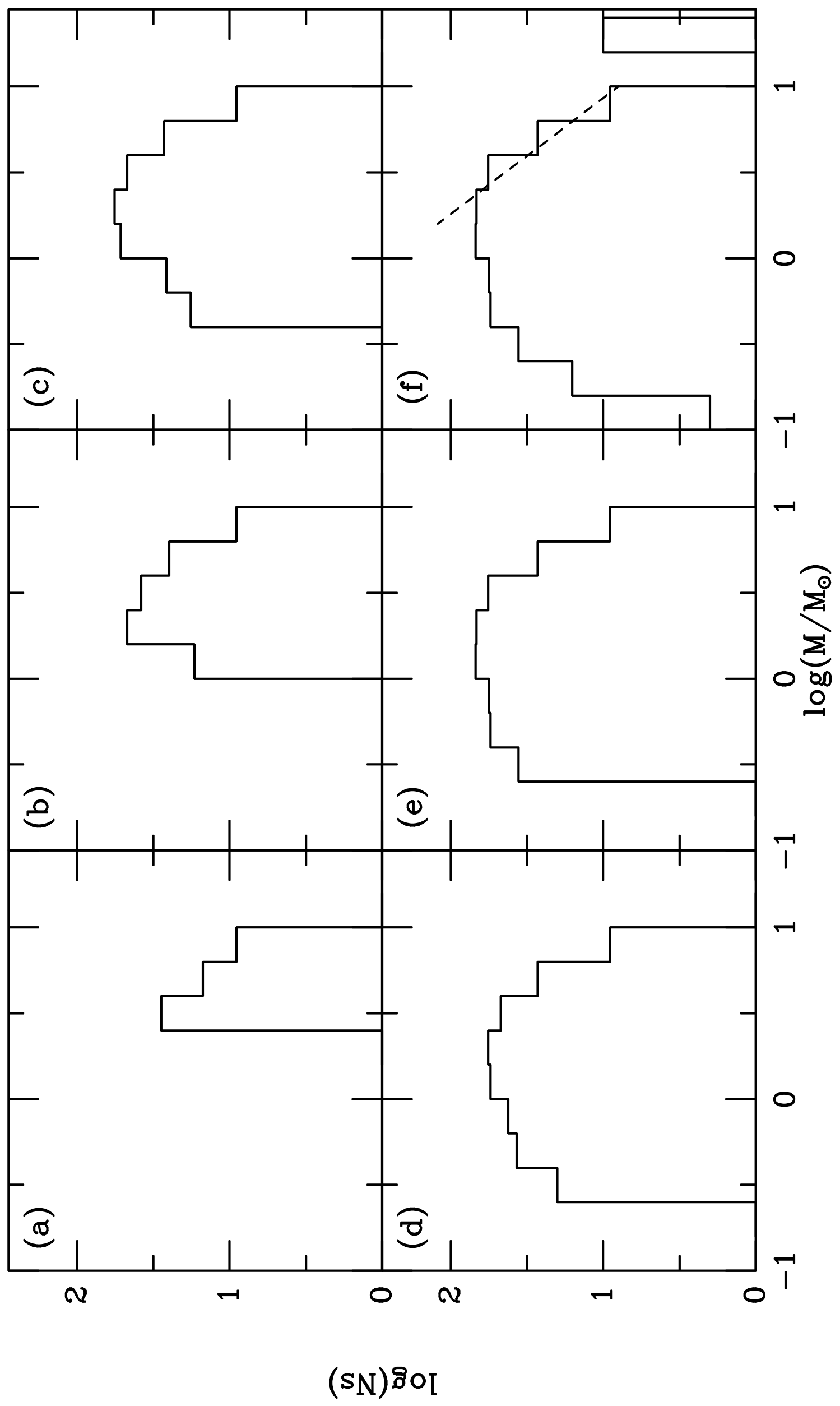




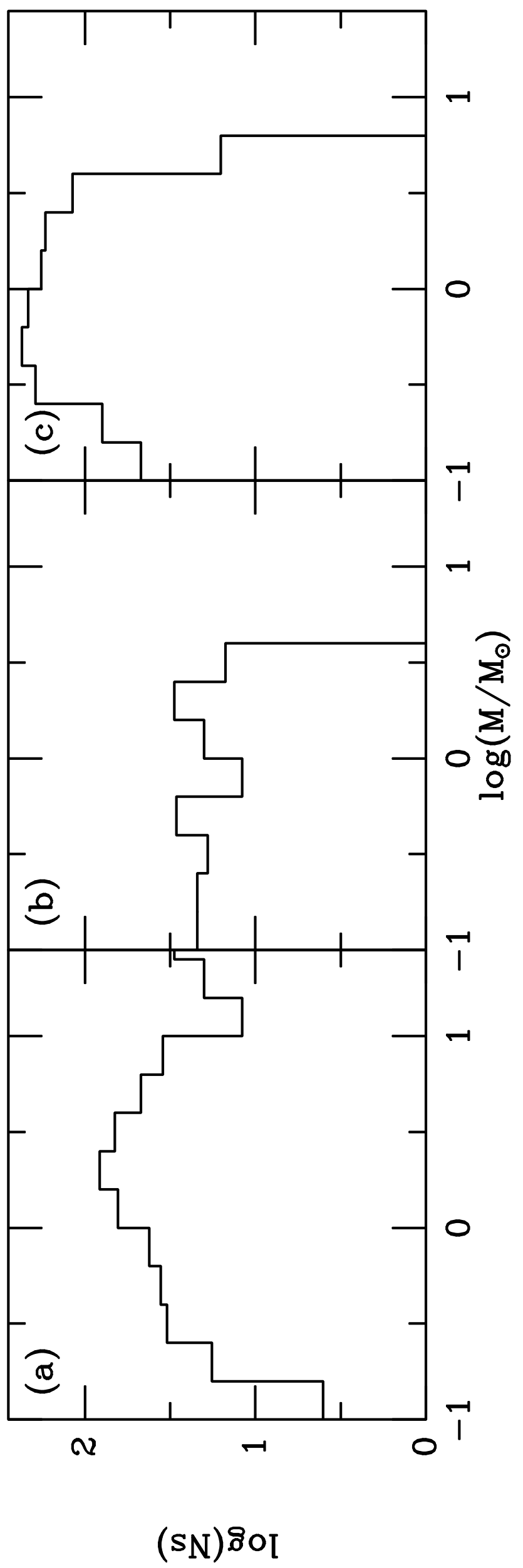




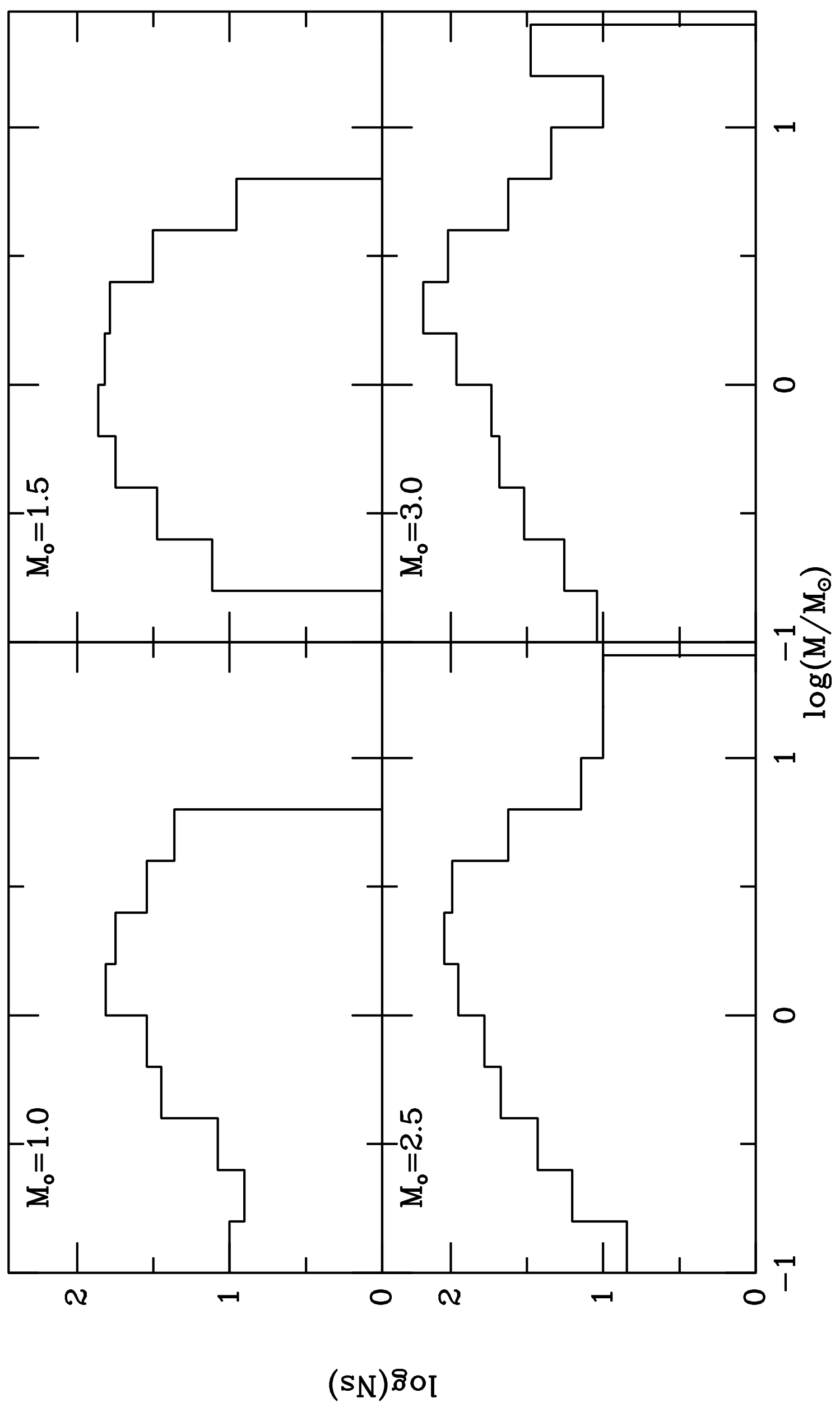




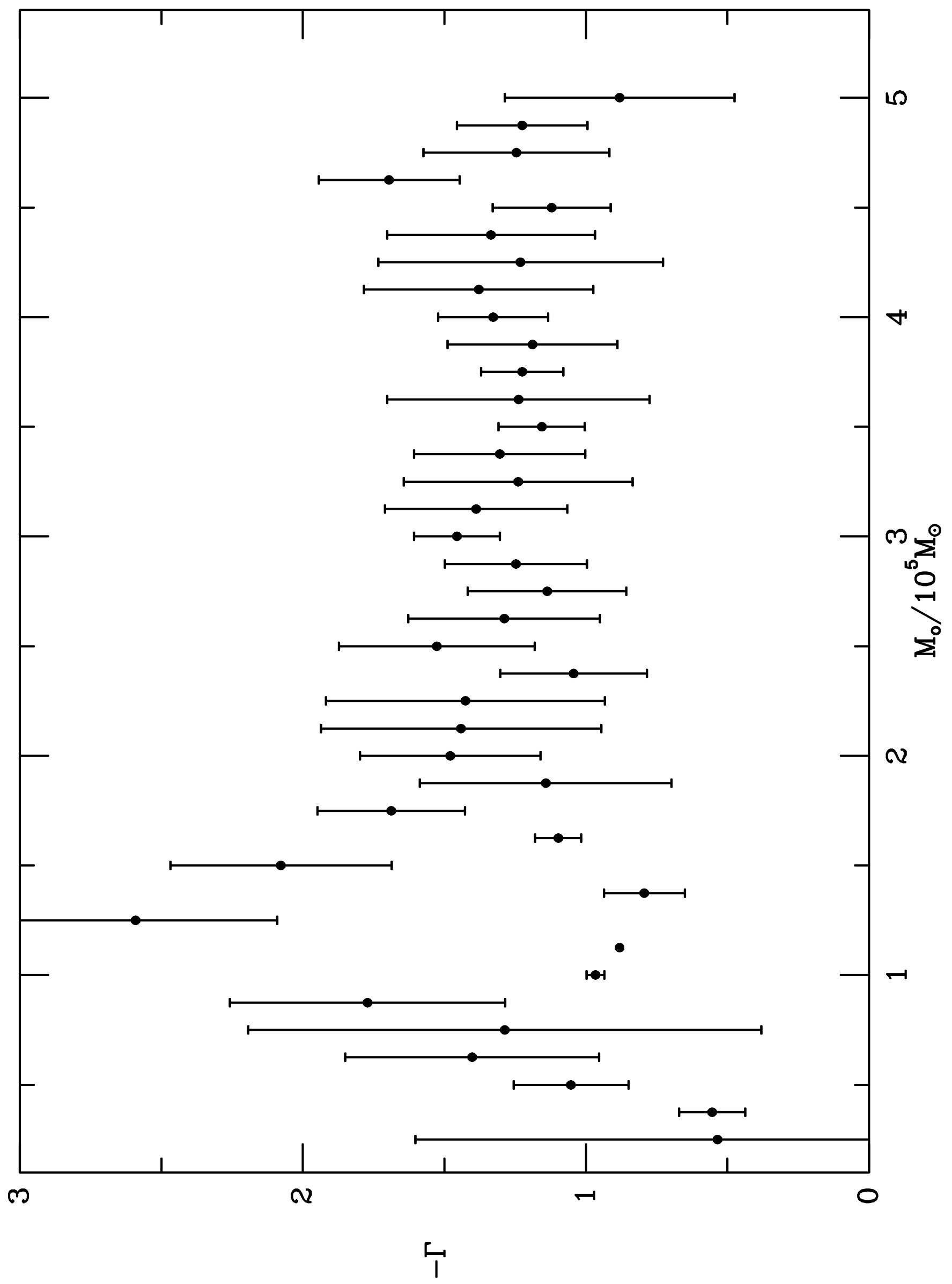




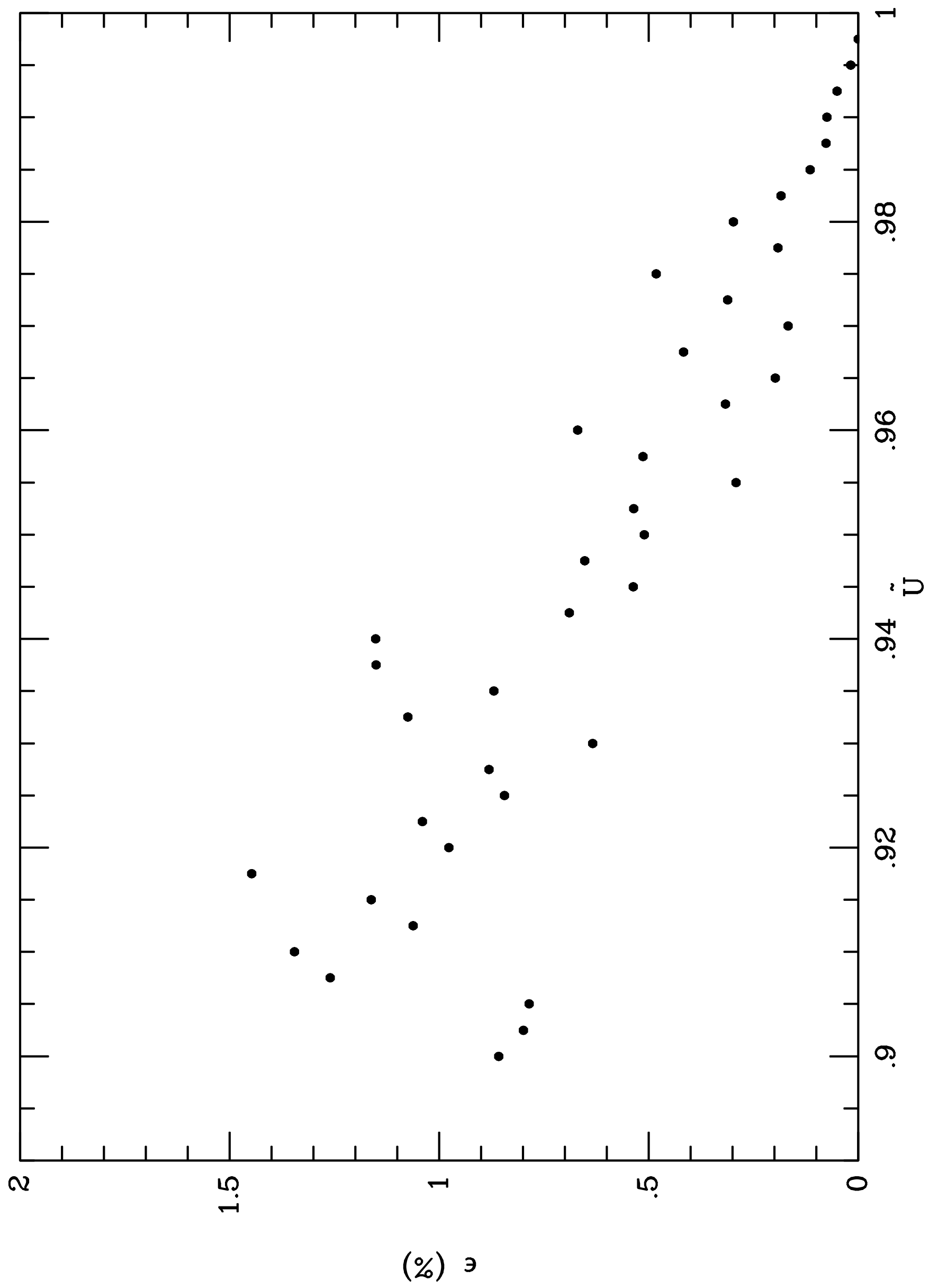




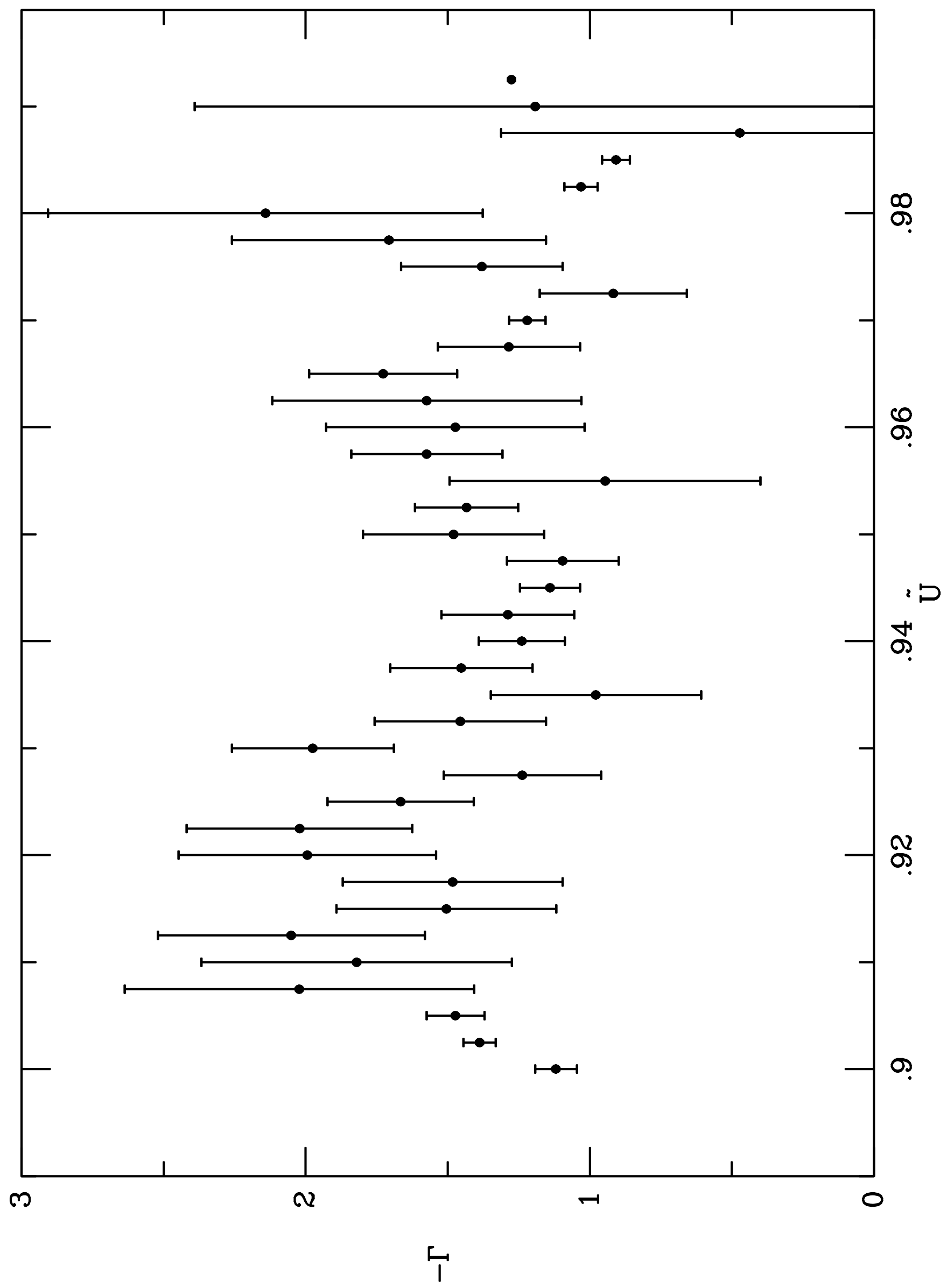




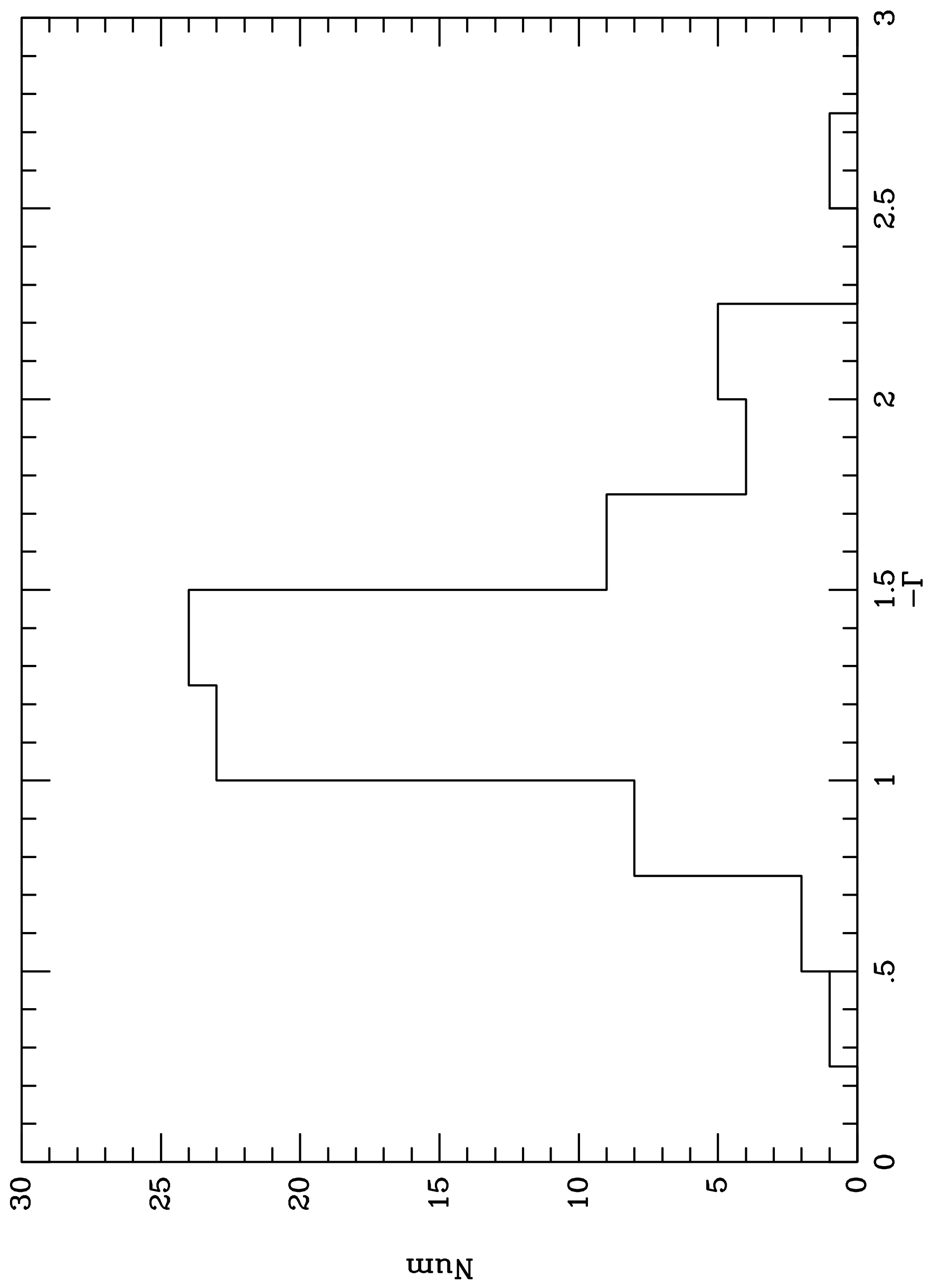

